\title{
SUPPLEMENTAL MATERIAL FOR MODELING THE EFFECT OF TEMPERATURE ON OZONE-RELATED MORTALITY
}

\author{
By Ander Wilson, Ana G. Rappold, Lucas M. Neas and Brian J. Reich \\ North Carolina State University, U.S. Environmental Protection Agency, U.S. Environmental \\ Protection Agency, and North Carolina State University
}

\section{APPENDIX A: ADDITIONAL FIGURES}

This section contains the additional figures referred to in the introduction and in Section 2.

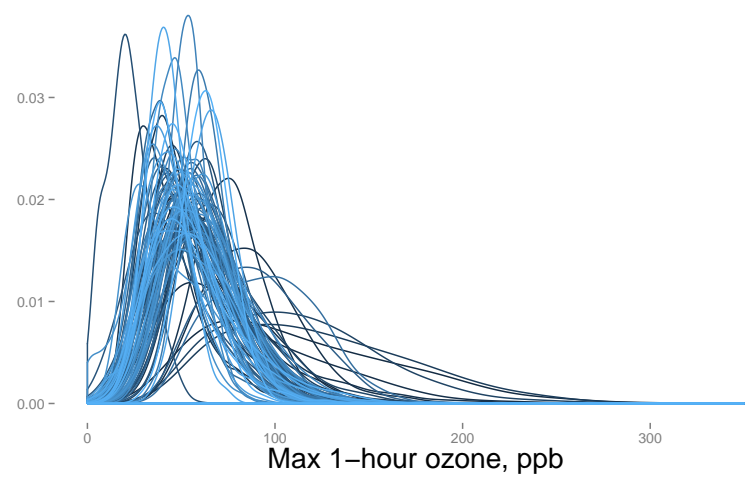

(a) Distribution of ozone by city.

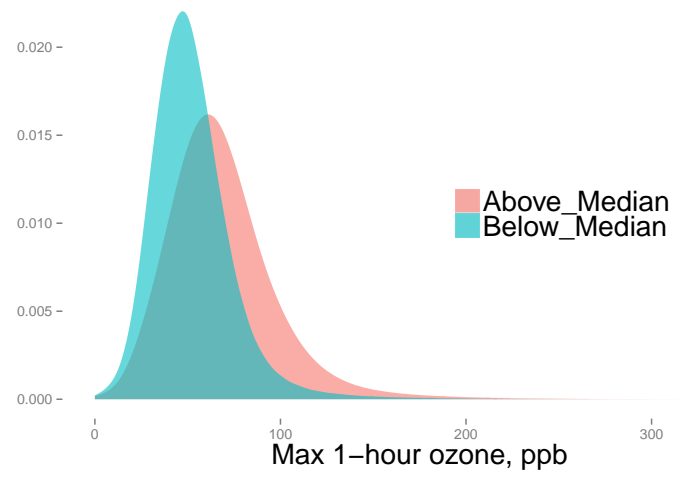

(c) Distribution of ozone on days above and below the cityspecific median temperature.

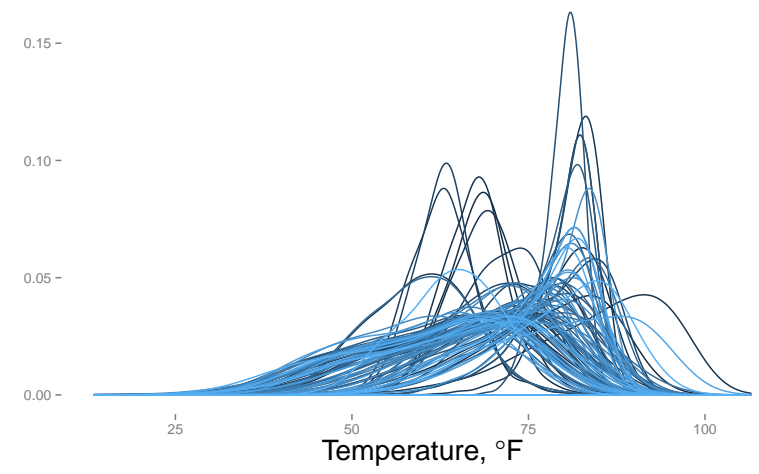

(b) Distribution of temperature by city.

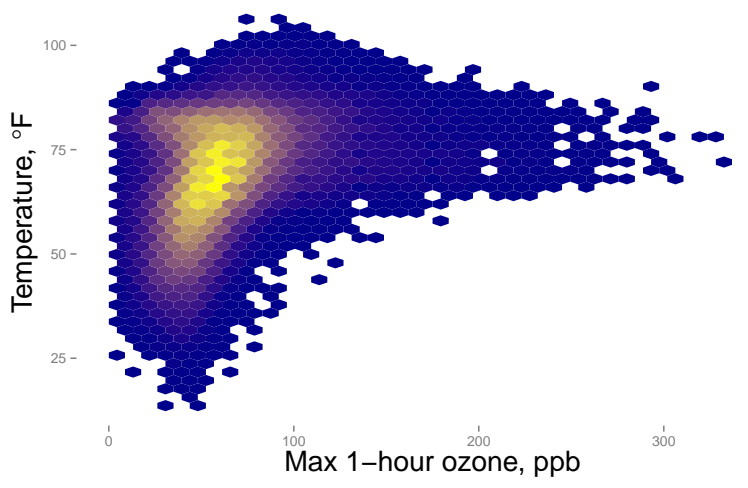

(d) National ozone-temperature distribution.

Fig 1: Summary of the ozone and temperature distribution. 


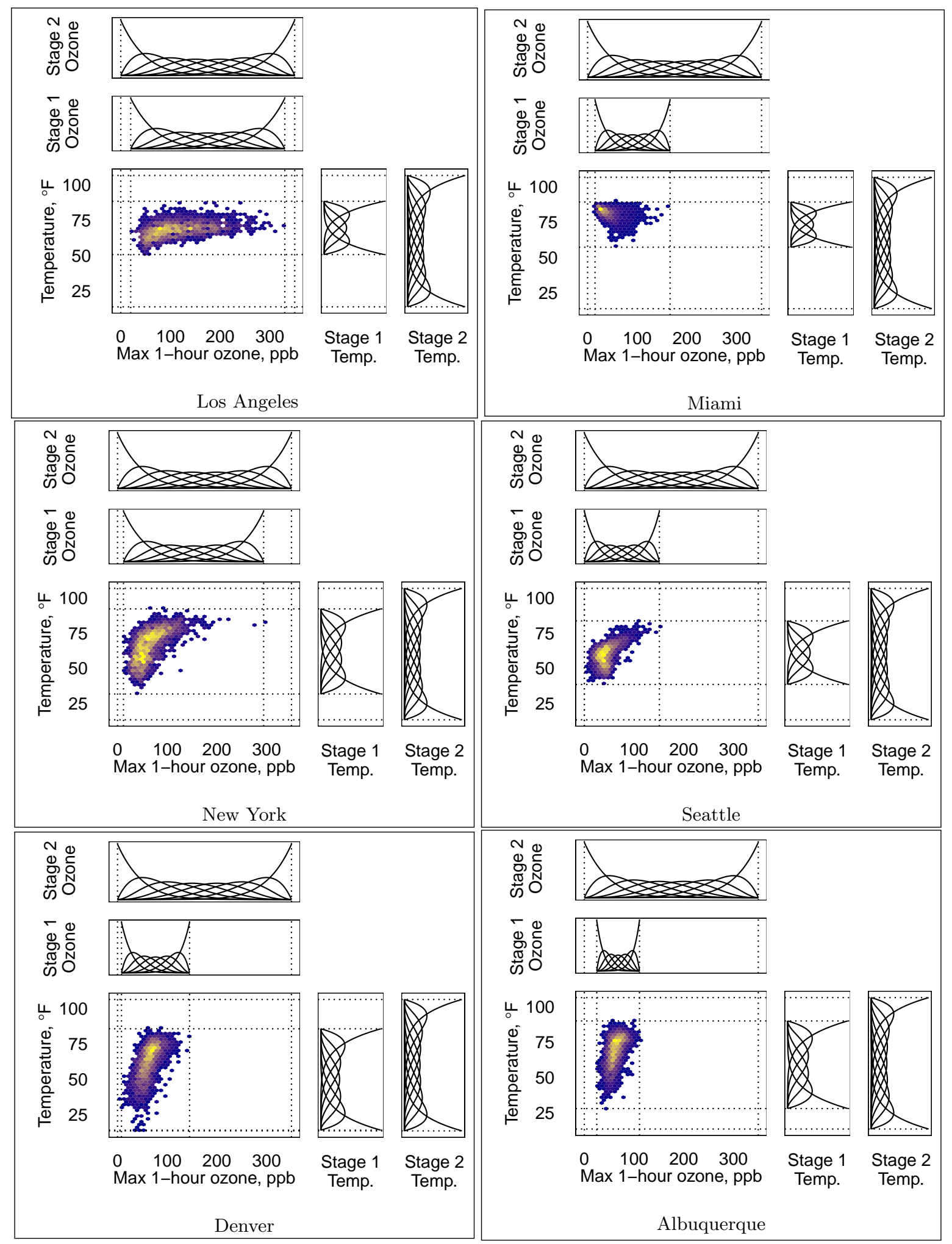

Fig 2: Demonstration of the different basis expansions used in the first and second stages. The bottom left shows the observed ozone-temperature distribution in each city. To the right are the basis functions in the temperature direction and to the top are the basis functions in the ozone direction for each stage. The first stage basis functions are different in each city. 


\section{APPENDIX B: CROSS-VALIDATION RESULTS}

This section contains the additional figures referred to in Section 3.1.

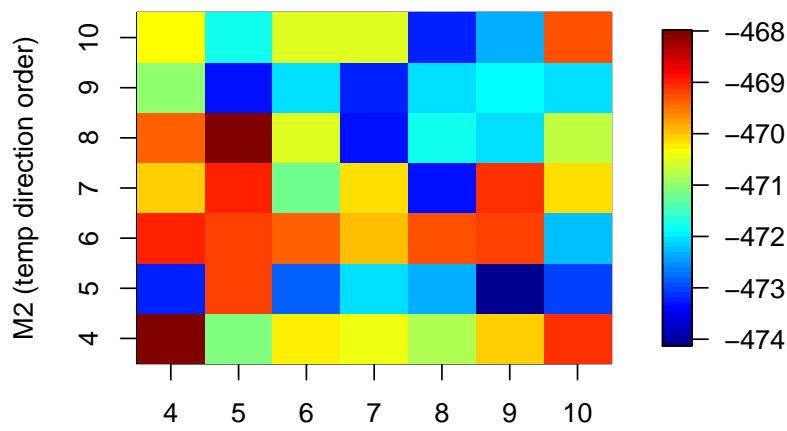

M1 (ozone direction order)

(a) Overall

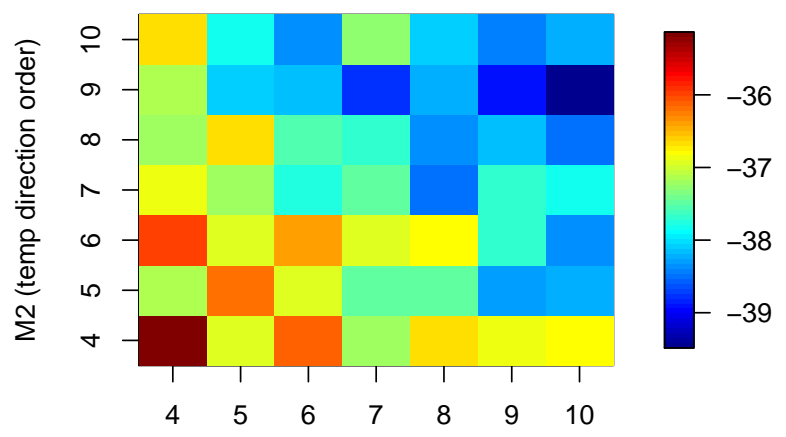

M1 (ozone direction order)

(c) Above 95th percentile of temperature

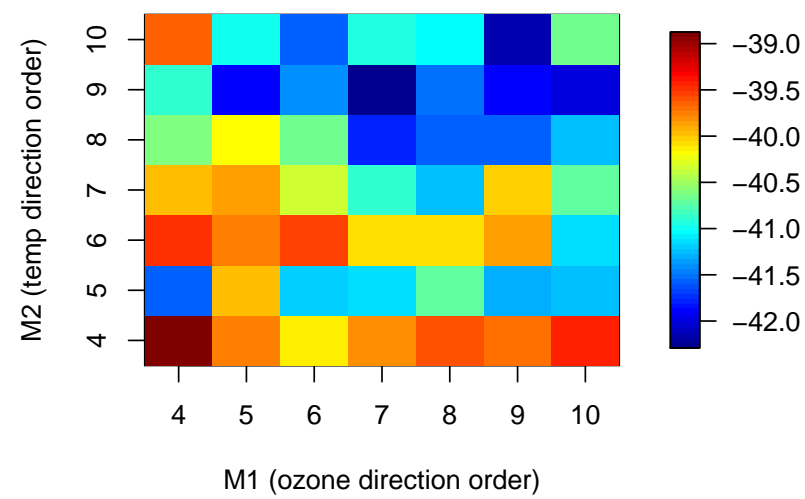

(b) Above 95th percentile of ozone

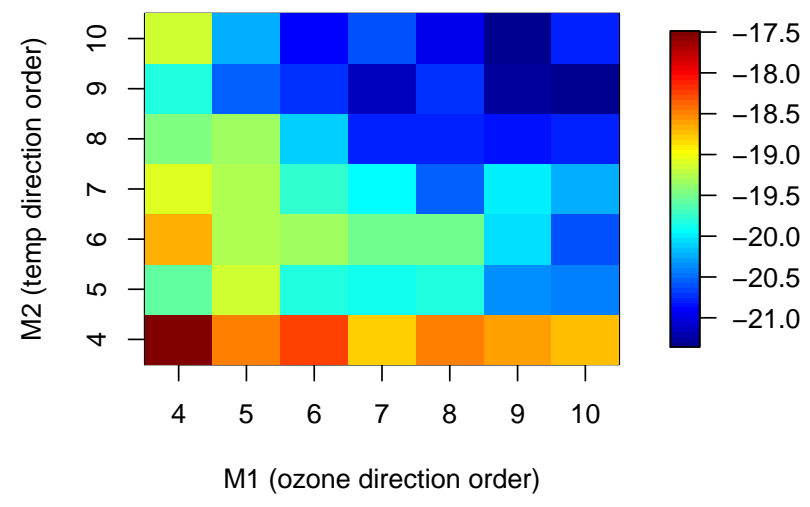

(d) Above 95th percentile of ozone and temperature

Fig 3: Difference in deviance from the linear, additive model (NMMAPS model) for the spatial monotone model with different numbers of basis functions in the ozone (M1) and temperature (M2) directions. 


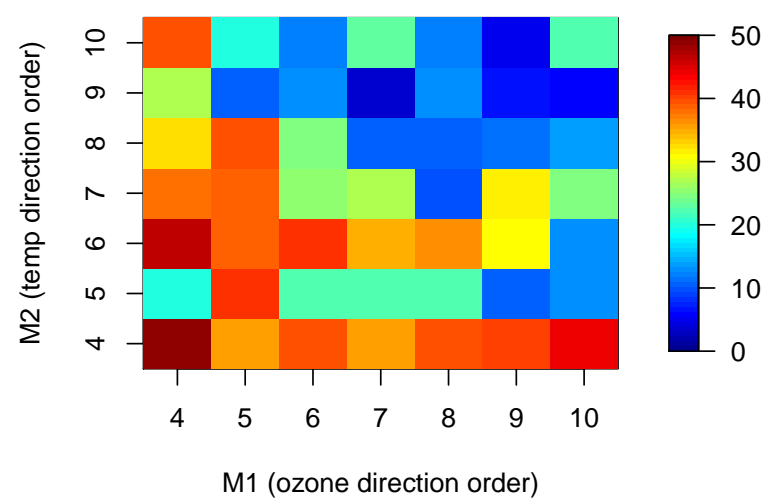

Fig 4: Average rank of CV results across Figures 3a through 3d, with one being the best performing model. The best model on average was $\left(M_{1}, M_{2}\right)=(7,9)$ with an average rank of 3.75 ; this is the model used for analysis. The next best models were $(9,10),(10,9)$, and $(9,9)$ with average ranks of $5.25,5.50$, and 6.75 , respectively.

\section{APPENDIX C: FULL CONDITIONAL DISTRIBUTION}

Let $p_{1}=M_{1}+1, p_{2}=M_{2}+1, p=p_{1} * p_{2}, \mathbf{S}=\mathbf{S}_{\mathbf{2}} \otimes \mathbf{S}_{\mathbf{1}}$. The posterior takes the form

$$
\begin{aligned}
L\left(\boldsymbol{\theta}, \boldsymbol{\mu}, \rho, \mathbf{S}_{\mathbf{2}}, \mathbf{S}_{\mathbf{1}} \mid \boldsymbol{\beta}_{c_{1}}, \ldots, \boldsymbol{\beta}_{c_{N}}, \mathbf{V}_{c_{1}}^{-1}, \ldots, \mathbf{V}_{c_{N}}^{-1}\right) \propto \\
\left.\quad \mathbf{S}_{\mathbf{2}}\right|^{-\left\{(N+1) p_{1}+p_{2}+1\right\} / 2}\left|\mathbf{S}_{\mathbf{1}}\right|^{-\left\{(N+1) p_{2}+p_{1}+1\right\} / 2}|\mathbf{R}|^{-p / 2} \tau^{-p / 2+a_{\tau}-1} \\
\quad \times \exp \left\{-\frac{1}{2} \operatorname{tr}\left\{\mathbf{S}_{\mathbf{2}}{ }^{-1}\right\}-\frac{1}{2} \operatorname{tr}\left\{\mathbf{S}_{\mathbf{1}}{ }^{-1}\right\}-\frac{1}{2 \tau}\left(\boldsymbol{\mu}-\mathbf{1}_{\mathbf{p}} \mu_{0}\right)^{T}(\mathbf{S})^{-1}\left(\boldsymbol{\mu}-\mathbf{1}_{\mathbf{p}} \mu_{0}\right)\right. \\
\quad-\frac{1}{2} \sum_{c}\left(\boldsymbol{\beta}_{c}-\mathbf{A}_{c} \boldsymbol{\theta}_{c}\right)^{T} \mathbf{V}_{c}^{-1}\left(\boldsymbol{\beta}_{c}-\mathbf{A}_{c} \boldsymbol{\theta}_{c}\right)-\frac{1}{2}\left(\boldsymbol{\theta}^{*}-\boldsymbol{\mu}\right)^{T}(\mathbf{R} \otimes \mathbf{S})^{-1}\left(\boldsymbol{\theta}^{*}-\boldsymbol{\mu}\right) \\
\left.-\frac{\left(\log \rho-\mu_{\rho}\right)^{2}}{2 \sigma_{\rho}^{2}}-\frac{\mu_{0}^{2}}{2 \tau_{0}}-b_{\tau} \tau^{-1}\right\} .
\end{aligned}
$$

Where $\boldsymbol{\theta}^{*}=\left(\boldsymbol{\theta}^{*}\left(c_{1}\right)^{T}, \ldots, \boldsymbol{\theta}^{*}\left(c_{N}\right)^{T}\right)^{T}$ and $\boldsymbol{\mu}=\left(\boldsymbol{\mu}\left(c_{1}\right)^{T}, \ldots, \boldsymbol{\mu}\left(c_{N}\right)^{T}\right)^{T}$ are $N p \times 1$ vectors.

\section{C.1. Full Conditionals.}

$$
\boldsymbol{\mu} \mid \text { rest } \sim \mathrm{N}\left\{\left(\frac{1}{\tau}+\mathbf{1}_{N}^{T} \mathbf{R}^{-1} \mathbf{1}_{N}\right)^{-1}\left(\mathbf{1}_{p} \frac{\mu_{0}}{\tau}+\sum_{c=1}^{N} \boldsymbol{\theta}_{c} \mathbf{R}_{c \cdot}^{-1} \mathbf{1}_{N}\right),\left(\frac{1}{\tau}+\mathbf{1}_{N}^{T} \mathbf{R}^{-1} \mathbf{1}_{N}\right)^{-1} \mathbf{S}\right\}
$$

(3) $\mu_{0} \mid$ rest $\sim \mathrm{N}\left\{\left(\tau^{-1} \mathbf{1}_{\mathbf{p}}^{\mathbf{T}} \mathbf{S}^{-1} \mathbf{1}_{\mathbf{p}}+\tau_{0}^{-1}\right)^{-1} \tau^{-1} \mathbf{1}_{\mathbf{p}}^{\mathbf{T}} \mathbf{S}^{-1} \boldsymbol{\mu},\left(\tau^{-1} \mathbf{1}_{\mathbf{p}}^{\mathbf{T}} \mathbf{S}^{-1} \mathbf{1}_{\mathbf{p}}+\tau_{0}^{-1}\right)^{-1}\right\}$

(4) $\tau^{-1} \mid$ rest $\sim \operatorname{Ga}\left\{a_{\tau}+p / 2, b_{\tau}+\left(\boldsymbol{\mu}-\mathbf{1}_{\mathbf{p}} \mu_{0}\right)^{T} \mathbf{S}^{-1}\left(\boldsymbol{\mu}-\mathbf{1}_{\mathbf{p}} \mu_{0}\right) / 2\right\}$

(5) $\mathbf{S}_{2} \mid$ rest $\sim \operatorname{IW}\left\{(N+1) p_{1}+p_{2}+1\right.$,

$$
\left.\mathbf{I}+(\widetilde{\boldsymbol{\theta}}-\tilde{\boldsymbol{\mu}})\left(\mathbf{R} \otimes \mathbf{S}_{1}\right)^{-1}(\widetilde{\boldsymbol{\theta}}-\tilde{\boldsymbol{\mu}})^{T}+\tau^{-1}\left(\tilde{\boldsymbol{\mu}}-\tilde{\boldsymbol{\mu}}_{0}\right) \mathbf{S}_{1}^{-1}\left(\widetilde{\boldsymbol{\mu}}-\tilde{\boldsymbol{\mu}}_{0}\right)^{T}\right\}
$$

(6) $\mathbf{S}_{1} \mid$ rest $\sim \operatorname{IW}\left\{(N+1) p_{2}+p_{1}+1\right.$,

$$
\left.\mathbf{I}+(\widetilde{\boldsymbol{\theta}}-\widetilde{\boldsymbol{\mu}})\left(\mathbf{R} \otimes \mathbf{S}_{2}\right)^{-1}(\widetilde{\boldsymbol{\theta}}-\widetilde{\boldsymbol{\mu}})^{T}+\tau^{-1}\left(\widetilde{\boldsymbol{\mu}}-\widetilde{\boldsymbol{\mu}}_{0}\right) \mathbf{S}_{2}^{-1}\left(\widetilde{\boldsymbol{\mu}}-\widetilde{\boldsymbol{\mu}}_{0}\right)^{T}\right\}
$$




$$
\theta_{j k, c} \mid \text { rest } \sim(1-\delta) \mathrm{TN}_{(-\infty, 0]}\left(0 ; E_{b}, V_{b}\right)+\delta \mathrm{TN}_{(0,-\infty)}\left(0 ; E_{a}, V_{a}\right)
$$

where

$$
\begin{aligned}
V_{a}= & {\left[\mathbf{S}_{1, j j}^{-1} \mathbf{S}_{2, k k}^{-1} \mathbf{R}_{c c}^{-1}+\left[\mathbf{A}_{\mathbf{c}}^{\mathbf{T}} \mathbf{V}_{\mathbf{c}}^{-\mathbf{1}} \mathbf{A}_{\mathbf{c}}\right]_{(j k),(j k)}\right]^{-1} } \\
E_{a}= & V_{a}\left[\mathbf{S}_{1, j j}^{-1} \mathbf{S}_{2, k k}^{-1} \mathbf{R}_{c c}^{-1} \mu_{j k}+\sum_{\mathcal{K}} \mathbf{S}_{1, j j^{\prime}}^{-1} \mathbf{S}_{2, k k^{\prime}}^{-1} \mathbf{R}_{c c^{\prime}}^{-1}\left(\mu_{j^{\prime} k^{\prime}}-\theta_{j^{\prime} k}^{*}\left(c^{\prime}\right)\right)\right. \\
& \left.+\left[\mathbf{A}_{\mathbf{c}}^{\mathbf{T}} \hat{\mathbf{V}}^{-1}\right]_{c^{\prime},(j k)[j k]}\left(\hat{\boldsymbol{\beta}}_{c^{\prime}[j k]}-\left(\mathbf{A}_{c} \boldsymbol{\theta}\left(c^{\prime}\right)\right)_{[j k]}\right)+\mathbf{A}_{\mathbf{c}}^{\mathbf{T}} \hat{\mathbf{V}}_{c,(j, k),(j, k)}^{-1} \hat{\boldsymbol{\beta}}_{c, j, k}\right] \\
V_{b}= & {\left[\mathbf{S}_{1, j j}^{-1} \mathbf{S}_{2, k k}^{-1} \mathbf{R}_{c c}^{-1}\right]^{-1} } \\
E_{b}= & V_{b}\left[\mathbf{S}_{1, j j}^{-1} \mathbf{S}_{2, k k}^{-1} \mathbf{R}_{c c}^{-1} \mu_{j k}+\sum_{\mathcal{K}} \mathbf{S}_{1, j j^{\prime}}^{-1} \mathbf{S}_{2, k k^{\prime}}^{-1} \mathbf{R}_{c c^{\prime}}^{-1}\left(\mu_{j^{\prime} k^{\prime}}-\theta_{j^{\prime} k}^{*}\left(c^{\prime}\right)\right)\right]
\end{aligned}
$$

and $\mathcal{K}=\left\{\left(j^{\prime}, k^{\prime}, c^{\prime}\right):\left\{j^{\prime} \neq j\right\} \cup\left\{k^{\prime} \neq k\right\} \cup\left\{c^{\prime} \neq c\right\}\right\}$. For the truncated parameters

$$
\delta=\frac{\frac{1-F\left(x ; E_{a}, V_{a}\right)}{f\left(x ; E_{a}, V_{a}\right)}}{\frac{F\left(x ; E_{b}, V_{b}\right)}{f\left(x ; E_{b}, V_{b}\right)}+\frac{1-F\left(x ; E_{a}, V_{a}\right)}{f\left(x ; E_{a}, V_{a}\right)}},
$$

and $\delta=1$ for unconstrained intercept, $j=0$.

The acceptance probability for the proposed range parameter $\rho^{\prime}$ proposed from $\rho^{\prime} \sim \mathrm{N}\left(\rho, s^{2}\right)$ centered on the current value $\rho$ with proposal variance $s^{2}$ is

$$
r_{\rho}=\frac{\left|\mathbf{R}^{\prime}\right|^{-p / 2} \exp \left\{-\frac{1}{2}\left(\boldsymbol{\theta}^{*}-\boldsymbol{\mu}\right)^{T}\left(\mathbf{R}^{\prime} \otimes \mathbf{S}\right)^{-1}\left(\boldsymbol{\theta}^{*}-\boldsymbol{\mu}\right)-\frac{\left(\log \rho^{\prime}-\mu_{\rho}\right)^{2}}{2 \sigma_{\rho}^{2}}\right\}}{|\mathbf{R}|^{-p / 2} \exp \left\{-\frac{1}{2}\left(\boldsymbol{\theta}^{*}-\boldsymbol{\mu}\right)^{T}(\mathbf{R} \otimes \mathbf{S})^{-1}\left(\boldsymbol{\theta}^{*}-\boldsymbol{\mu}\right)-\frac{\left(\log \rho-\mu_{\rho}\right)^{2}}{2 \sigma_{\rho}^{2}}\right\}},
$$

where $\mathbf{R}$ is the current correlation matrix and $\mathbf{R}^{\prime}$ is the proposed correlation matrix. 


\section{APPENDIX D: MCMC ALGORITHM}

The MCMC algorithm proceeds as follows.

Step 1: Generate a random integer $r$ from a geometric distribution with mean $\left(1-\epsilon_{\rho}\right) / \epsilon_{\rho}$. Propose a new $\log \rho$ from a normal and accept the proposed values with probability (13) $r$ times.

Step 2: For each city in $c=1, \ldots, n$ do the following:

Step2a: Generate a random integer $r$ from a geometric distribution with mean $\left(1-\epsilon_{\theta}\right) / \epsilon_{\theta}$.

Step2b: Do the following $r$ times.

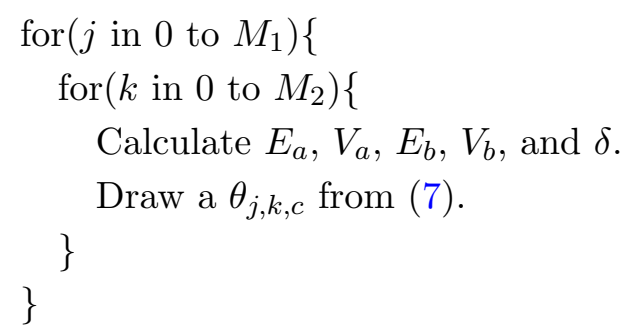

Step 3: Update $\mathbf{S}_{1}$ and $\mathbf{S}_{2}$ from (6) and (7).

Step 4: Update $\boldsymbol{\mu}$ from (2).

Step 5: Update $\mu_{0}$ from (3).

Step 6: Update $\boldsymbol{\mu}$ from (4).

In steps 1 and 2, we choose a random number of updates for the range and for each city's basis coefficients. This approach substantially reduced autocorrelation and improves performance. We used $\epsilon_{\rho}=0.8$ and $\epsilon_{\theta}=0.9$.

\section{APPENDIX E: TRACE PLOTS}

This section contains a random sample of trace plots as well as the trace plot for the range. 

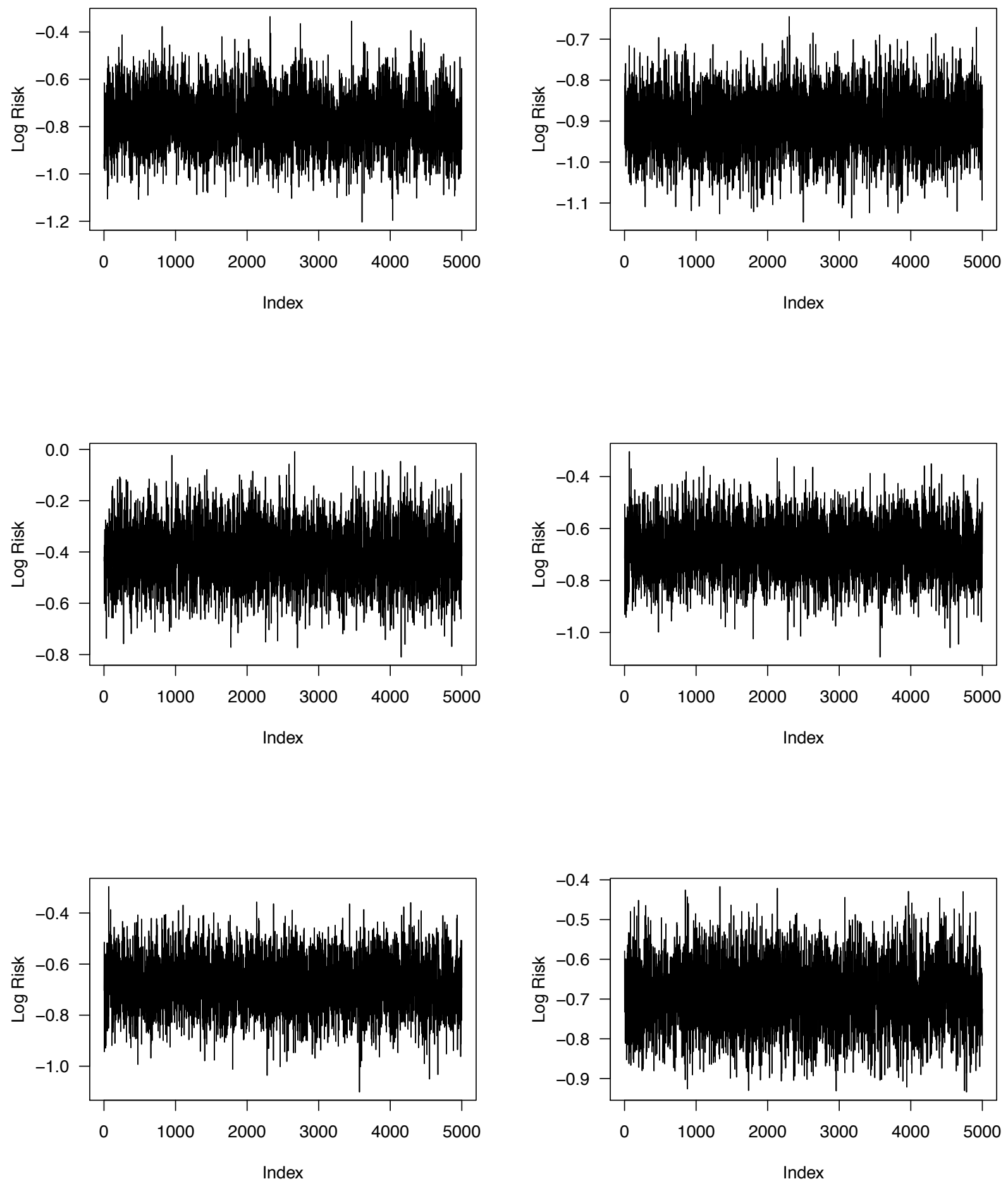

Fig 5: Trace plots of the point wise estimates of the risk surface. 


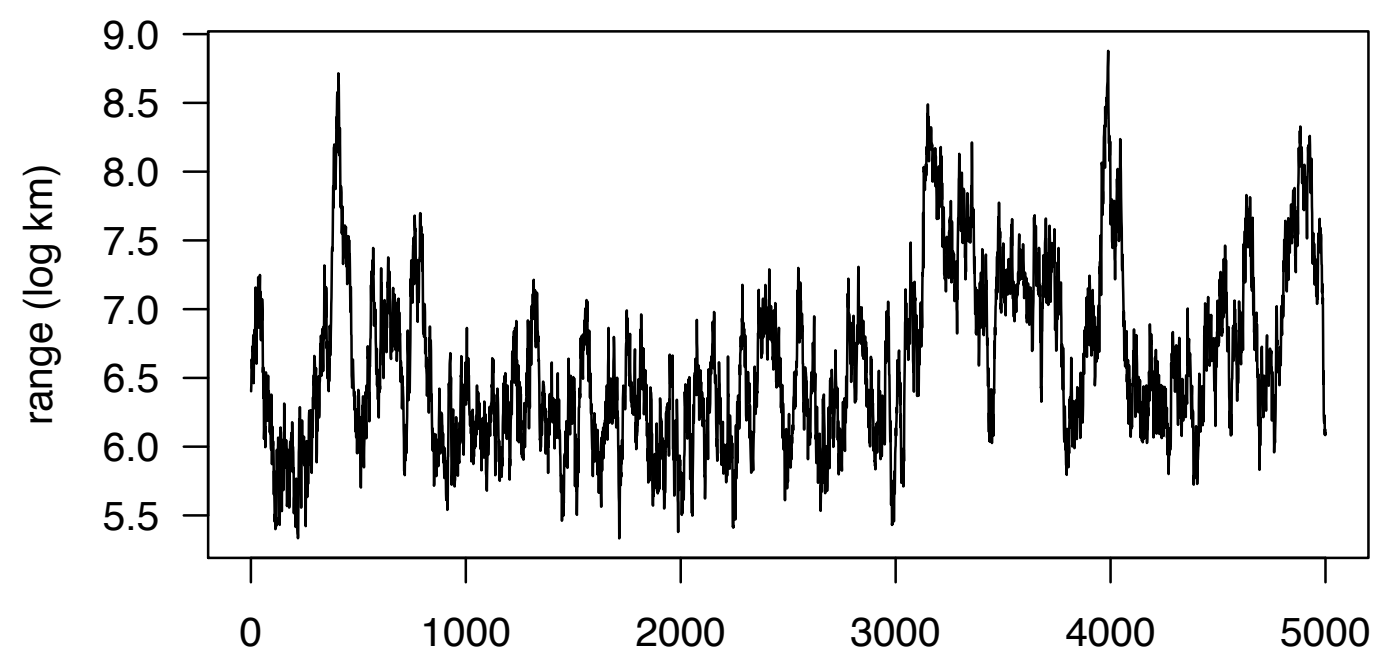

Fig 6: Trace of the posterior sample of $\log \rho$ (log range in $\log$ kilometers). The trace plot only includes the posterior sample and does not show the portion of the burn in portion of the chain. The posterior mean is 922.9 , the median is 659.4 , and the $95 \%$ symmetric interval is $(291.8,3134.5)$.

\section{REFERENCES}

AdDress of the First and Fourth Authors

Department of Statistics

North Carolina State University

2311 Stinson Drive

Raleigh, North Carolina, 27695, U.S.A.

E-MAIL: ander_wilson@ncsu.edu

brian_reich@ncsu.edu
Address of the Second And Third authors Environmental Public Health Division U.S Environmental Protection Agency Chapel Hill, NC 27599

E-MAIL: rappold.ana@epa.gov neas.lucas@epa.gov 